\title{
RAMAN SCATTERING IN PSEUDOSPIN-ELECTRON MODEL
}

\author{
I. V. Stasyuk, T. S. Mysakovych \\ Institute for Condensed Matter Physics of the National Academy of Sciences of Ukraine, \\ 1 Svientsitskii Str., Lviv, UA-79011, Ukraine
}

(Received September 2, 1999)

\begin{abstract}
Anharmonic phonon (pseudospin) contributions to Raman light scattering in the crystals described by the pseudospin-electron model with the strong short-range electron correlation are investigated. The microscopic approach based on the operator expansion of the polarizability operator in powers of the electron transfer constant is used for calculation of the components of the Raman scattering tensor. The influence of the effective interaction between pseudospins via conducting electrons is taken into account in the framework of generalized random phase approximation. The features of the collective pseudospin dynamics are investigated; the scattering spectrum components connected with excitations of the pseudospin-wave type as well as with the reconstruction of electron spectrum at the pseudospin reorientation are separated. The frequency dependence of the Raman light scattering intensity is analyzed at various values of electron concentration and temperature.
\end{abstract}

Key words: Raman scattering, pseudospin-electron model, strong electron correlation.

PACS numbers: 72.10.-d, 72.10.Di

\section{INTRODUCTION}

Due to the discovery of the phenomenon of hightemperature superconductivity the increasing attention of researchers has been paid in the last few years to the investigation of crystals with the strong electron short-range correlations. The high- $T_{c}$ superconductors (HTSC) belong to this class of objects. Besides the very effect of superconducting paring of electrons the characteristic property of these materials is the possibility of antiferromagnetic ordering, the strong lattice anharmonicity having local character, the existence of phase transitions and instabilities caused by the interaction of electrons with vibrational degrees of freedom. Because of that many known physical effects become peculiar in these crystals. Their mechanisms are more complicated, the new ones appearing in addition.

In this respect the Raman light scattering phenomenon can serve as a characteristic example. It is connected usually with a scattering on phonon vibrations. In crystals with strongly correlated electrons the new mechanism of this effect appears: the electron scattering due to the transitions between electron band states [1]. Moreover, owing to the antiferromagnetic state appearance under the influence of the electron Hubbard correlation (the $t-J$ model) the scattering on antiferromagnetic magnons becomes possible (the two-magnon scattering process considered for the first time by Fleury and Loudon [2]). Such contributions to the Raman scattering cross-section were considered on a microscopic level by Shastry and Shraiman [3] for the case of electron systems described by the Hubbard model.

At the same time, experimental investigations of frequency dependences of the Raman scattering intensity in HTSC crystals of the $\mathrm{YBa}_{2} \mathrm{Cu}_{3} \mathrm{O}_{7-\delta}$ group revealed (at the oxygen vacancies content corresponding to the antiferromagnetic phase region) significant deviations of the observed Raman line profiles from the predicted ones by the electron and, especially, magnon mechanisms of scattering [4]. The noticeable asymmetry of lines, that is connected with the existence of broad wings from the side of large frequency values, can be mentioned in this context. Allowance for another factors (such as the electronphonon interaction), that can influence these scattering processes, requires a widening of description and going beyond the purely electron models.

The step in this direction was made in [5], where the main contributions to the Raman scattering crosssection in the pseudospin-electron model (PEM) were considered on the basis of microscopic approach [6,7] and its generalization given in [8] (where the operator expansion scheme at the construction of the polarizability operator of a system was proposed). The PEM model was developed with the purpose of allowance for the presence of locally anharmonic elements of the crystal structure in the systems of the $\mathrm{YBa}_{2} \mathrm{Cu}_{3} \mathrm{O}_{7-\delta}$ type; the description of dynamics of such structure fragments is performed in this model by means of the pseudospin formalism $[9,10]$. It was established that the pseudospin-electron interaction of the contact type $\left(\sim g S_{i}^{z} n_{i}\right)$ leads to an additional splitting in the electron spectrum. This makes the picture of transitions at the electron and magnon scattering more rich and complicated. In addition to this a new mechanism of scattering appears in the presence of the transverse field acting on pseudospins (that describes the tunneling of particle moving in the double-well potential). Namely, the scattering on the local anharmonic vibrations (the pseudospin reorientation) modified by the interaction with conducting electrons becomes possible in this case.

This work is devoted to a more detailed investigation of the pseudospin mechanism of Raman scattering in PEM. The corresponding contributions to the polarizability op- 
erator and on this basis to the Raman scattering tensor will be separated. The calculations of the pseudospin correlation functions that appear in the corresponding expressions will be performed allowing for the effective retarded interaction between pseudospins via conducting electrons. We will base on the generalized random phase approximation (GRPA) that was proposed for a calculation of boson correlation functions and susceptibilities in the case of the models with strongly correlated electrons (spin correlators and magnetic susceptibility for the Hubbard and $t-J$ models [11]; density and charge correlators and dielectric susceptibility for PEM with zero transverse field [12]). The GPRA can be treated as analogue of the usual random phase approximation for electron systems, but at the basic allowance for the short-range electron correlations.

We will analyse the frequency dependences of the scat- tering intensity at various relations between parameters of the theory and at the certain values of the electron concentration and temperature. The factors which determine the form of the line profiles in the case of the pseudospin Raman scattering will be studied. On the basis of the obtained results the main features of the collective pseudospin dynamics will be discussed.

\section{SCATTERING TENSOR FOR PSEUDOSPIN-ELECTRON MODEL}

In our approach to the description of Raman light scattering we start from the expression for the crosssection of scattering written in terms of the time correlation function calculated on the polarizability operators $\hat{P}[6,7]$ :

$$
\frac{\partial^{2} \sigma}{\partial \Omega \partial \omega_{2}}=\frac{1}{\left(4 \pi \varepsilon_{0}\right)^{2}} \sqrt{\frac{\varepsilon_{1}}{\varepsilon_{2}}} \frac{\omega_{2}^{3} \omega_{1}}{\hbar^{2} c^{4}} \sum_{\alpha \beta \alpha^{\prime} \beta^{\prime}} e_{1 \alpha} e_{2 \beta} e_{1 \alpha^{\prime}} e_{2 \beta^{\prime}} H_{k_{2}, k_{1}}^{\beta^{\prime} \alpha^{\prime}, \beta, \alpha}\left(\omega_{1}, \omega_{2}\right)
$$

here $\mathbf{e}_{1}, \mathbf{e}_{2}$ are polarization vectors; $\omega_{1}, \omega_{2}$ are incident and scattered light frequencies; $\mathbf{k}_{1}, \mathbf{k}_{2}$ are corresponding wave vectors; $\varepsilon_{1,2} \equiv \varepsilon\left(\omega_{1}, \omega_{2}\right)$;

$H_{k_{2}, k_{1}}^{\beta^{\prime} \alpha^{\prime}, \beta, \alpha}\left(\omega_{1}, \omega_{2}\right)$ is the Raman scattering tensor:

$$
H_{k_{2},-k_{1} ;-k_{2}, k_{1}}^{\beta^{\prime} \alpha^{\prime}, \beta, \alpha}\left(\omega_{1}, \omega_{2}\right)=\frac{1}{2 \pi} \int_{-\infty}^{+\infty} d t e^{i\left(\omega_{1}-\omega_{2}\right) t}\left\langle\hat{P}_{\mathbf{k}_{2}-\mathbf{k}_{1}}^{\beta^{\prime} \alpha^{\prime}}\left(-\omega_{1}, t\right) \hat{P}_{-\mathbf{k}_{2} \mathbf{k}_{1}}^{\beta \alpha}\left(\omega_{1}, 0\right)\right\rangle
$$

$\hat{P}$ is the polarizability operator

$$
\hat{P}_{\mathbf{k}^{\prime} \mathbf{k}}^{\beta \alpha}(\omega, t)=-\int_{-\infty}^{+\infty} d s e^{i \omega(t-s)}\left\{\left\{\hat{M}^{\beta}\left(\mathbf{k}^{\prime}, t\right) \mid \hat{M}^{\alpha}(\mathbf{k}, s)\right\}\right\}
$$

here $\hat{M}^{\alpha}(\mathbf{k})$ is a Fourier transform of a dipole momentum of a crystal unit cell. The symbol $\left\{\left\{\hat{M}^{\beta}\left(\mathbf{k}^{\prime}, t\right) \mid \hat{M}^{\alpha}(\mathbf{k}, s)\right\}\right\}$ stands for "unaveraged" Green's function defined in the following way [8]:

$$
\left\{\left\{A(t) \mid B\left(t^{\prime}\right)\right\}\right\}=-i \Theta\left(t-t^{\prime}\right)\left[A(t), B\left(t^{\prime}\right)\right]
$$

where operators are written in Heisenberg representation. The equations of motion for this function have the form

$$
\begin{aligned}
& \omega_{1}\{\{A \mid B\}\}_{\omega_{1}, \omega_{2}}=\frac{1}{2 \pi}[A, B]_{\omega_{1}-\omega_{2}}+\{\{[A, H] \mid B\}\}_{\omega_{1}, \omega_{2}}, \\
& \omega_{2}\{\{A \mid B\}\}_{\omega_{1}, \omega_{2}}=\frac{1}{2 \pi}[A, B]_{\omega_{1}-\omega_{2}}-\{\{A \mid[B, H]\}\}_{\omega_{1}, \omega_{2}},
\end{aligned}
$$

where

$$
\{\{A \mid B\}\}_{\omega_{1}, \omega_{2}}=\frac{1}{(2 \pi)^{2}} \int_{-\infty}^{\infty} d t \int_{-\infty}^{+\infty} d s e^{i \omega_{1} t} e^{-i \omega_{2} s}\{\{A(t) \mid B(s)\}\}, \quad A_{\omega}=\frac{1}{2 \pi} \int_{-\infty}^{+\infty} d t e^{i \omega t} A(t)
$$




\section{V. STASYUK, T. S. MYSAKOVYCH}

The solution of equations (5) are built (as was proposed in [8]) in the form of operator series in the powers of certain parameters of the system Hamiltonian. In the case of models with strong short-range electron correlations the electron transfer constant $t_{i j}$ can be chosen as a formal expansion parameter. Such a procedure has been used in $[5,13]$. The Raman scattering tensor constructed in [3] in the framework of the effective scattering Hamiltonian approach was also obtained in the form of a similar expansion. [10]:

The Hamiltonian of the model has the following form

$$
H=\sum_{i} H_{i}+\sum_{i, j, \sigma} t_{i, j} \hat{c}_{i, \sigma}^{\dagger} \hat{c}_{j, \sigma}
$$

where the single-site term

$$
\begin{aligned}
H_{i} & =U n_{i \uparrow} n_{i \downarrow}-\mu\left(n_{i \uparrow}+n_{i \downarrow}\right) \\
& +g\left(n_{i \uparrow}+n_{i \downarrow}\right) S_{i}^{z}-\Omega S_{i}^{x}-h S_{i}^{z}
\end{aligned}
$$

describes the interaction of electrons with the local anharmonic vibrational modes represented by pseudospins ( $g$-term); the tunneling splitting of the vibrational mode $(\Omega$-term), the asymmetry of local potential described by longitudinal $h$ field and the Hubbard electron correlation $(U$-term). The second term in (6) is responsible for the electron transfer.

It is useful to introduce the following single-site basis of states $|i, R>\equiv| n_{i \uparrow}, n_{i \downarrow}, S_{i}^{z}=\frac{1}{2}>, \mid i, \widetilde{R}>\equiv$ $\mid n_{i \uparrow}, n_{i \downarrow}, S_{i}^{z}=-\frac{1}{2}>[10]$ :

$$
\begin{aligned}
& |i, 1>=| i, 0,0, \frac{1}{2}>, \quad|i, \widetilde{1}\rangle=\left|i, 0,0,-\frac{1}{2}\right\rangle, \\
& |i, 2>=| i, 1,1, \frac{1}{2}>, \quad|i, \widetilde{2}\rangle=\left|i, 1,1,-\frac{1}{2}\right\rangle, \\
& \left.|i, 3>=| i, 0,1, \frac{1}{2}>, \quad|i, \widetilde{3}>=| i, 0,1,-\frac{1}{2}\right\rangle, \\
& |i, 4>=| i, 1,0, \frac{1}{2}>, \quad|i, \widetilde{4}>=| i, 1,0,-\frac{1}{2}>
\end{aligned}
$$

The Hamiltonian $H_{i}$ can be reduced to a diagonal form with the help of the transformation

$$
\begin{aligned}
& \left|i, R>=\cos \phi_{r}\right| i, r>+\sin \phi_{r} \mid i, \widetilde{r}>, \\
& \left|i, \widetilde{R}>=\cos \phi_{r}\right| i, \widetilde{r}>-\sin \phi_{r} \mid i, r>
\end{aligned}
$$

where

$$
\begin{aligned}
\cos 2 \phi_{1} & =\frac{-h}{\sqrt{h^{2}+\Omega^{2}}} \\
\cos 2 \phi_{2} & =\frac{2 g-h}{\sqrt{(2 g-h)^{2}+\Omega^{2}}} \\
\cos 2 \phi_{3} & =\cos 2 \phi_{4}=\frac{g-h}{\sqrt{(g-h)^{2}+\Omega^{2}}}
\end{aligned}
$$

In terms of the operators $X_{i}^{r s}=|i, r><i, s|$ :

$$
H_{i}=\sum_{r} \lambda_{r} X_{i}^{r r}
$$

with

$$
\begin{aligned}
& \lambda_{1, \tilde{1}}= \pm \frac{1}{2} \sqrt{h^{2}+\Omega^{2}}, \\
& \lambda_{2, \tilde{2}}=-2 \mu+U \pm \frac{1}{2} \sqrt{(2 g-h)^{2}+\Omega^{2}} \\
& \lambda_{3, \tilde{3}}=\lambda_{4, \tilde{4}}=-\mu \pm \frac{1}{2} \sqrt{(g-h)^{2}+\Omega^{2}} .
\end{aligned}
$$

The total Hamiltonian is given in the Hubbard operators representation by the expression

$$
H=\sum_{i, r} \lambda_{r} X_{i}^{r r}+\sum_{i, j, \sigma} t_{i, j} \hat{c}_{i, \sigma}^{\dagger} \hat{c}_{j, \sigma}
$$

where

$$
\begin{aligned}
\hat{c}_{i, \uparrow}^{\dagger} & =\cos \left(\phi_{4}-\phi_{1}\right)\left(X_{i}^{41}+X_{i}^{\widetilde{41}}\right)-\sin \left(\phi_{4}-\phi_{1}\right)\left(X_{i}^{4 \tilde{1}}-X_{i}^{\tilde{4} 1}\right)+\cos \left(\phi_{2}-\phi_{3}\right)\left(X_{i}^{23}+X_{i}^{\widetilde{23}}\right) \\
& -\sin \left(\phi_{2}-\phi_{3}\right)\left(X_{i}^{2 \widetilde{3}}-X_{i}^{\widetilde{2} 3}\right), \\
\hat{c}_{i, \downarrow}^{\dagger} & =\cos \left(\phi_{3}-\phi_{1}\right)\left(X_{i}^{31}+X_{i}^{\widetilde{31}}\right)-\sin \left(\phi_{4}-\phi_{1}\right)\left(X_{i}^{3 \tilde{1}}-X_{i}^{\tilde{3} 1}\right)-\cos \left(\phi_{2}-\phi_{4}\right)\left(X_{i}^{24}+X_{i}^{\widetilde{24}}\right) \\
& +\sin \left(\phi_{2}-\phi_{4}\right)\left(X_{i}^{2 \widetilde{4}}-X_{i}^{\widetilde{2} 4}\right) .
\end{aligned}
$$


The dipole momentum of a unit cell is a sum of electronic and ionic( pseudospin) parts

$$
M_{i}^{\alpha}=e R_{i}^{\alpha}\left(n_{\uparrow, i}+n_{\downarrow, i}\right)+\frac{d_{s}}{2} S_{i}^{z} \delta_{\alpha \gamma},
$$

(the local anharmonic double well is oriented along the direction $\gamma$ ). For the layered crystal with the electron transfer only within the layers the problem of Raman scattering in a two-dimensional structure can be considered separately. In the case when the direction of local vibrations is perpendicular to the layer the transverse component of the vector $\mathbf{M}_{\mathbf{i}}$ can be written in the form $[10]$

$$
M_{i}^{\gamma}=d_{e}\left(n_{\uparrow, i}+n_{\downarrow, i}\right)+\frac{d_{s}}{2} S_{i}^{z}
$$

here $d_{e}$ is an effective dipole momentum, arising due to nonhomeopolarity of the filling of electron states on a site.

To calculate unaveraged Green's function $\left\{\left\{M_{k}^{\gamma} \mid M_{l}^{\gamma}\right\}\right\}$ which is necessary for the construction of the polarizability operator, we make an operator expansion, using the transfer constant $t_{i j}$ as a formal small parameter [5,13]. Using the equation of motion (5) we obtain in the zero order with respect to the parameter $t_{i j}$ :

$$
\begin{aligned}
\left\{\left\{M_{k}^{\gamma} \mid M_{l}^{\gamma}\right\}\right\}_{\omega_{2}, \omega_{1}} & =\frac{\delta_{k, l} d_{s}{ }^{2}}{4 \omega_{1}} \sum_{r} \sin 4 \phi_{r}\left(X_{k}^{r \tilde{r}}-X_{k}^{\tilde{r} r}\right)_{\omega_{2}-\omega_{1}} \\
& +\sum_{r} \frac{\delta_{k, l} d_{s}{ }^{2}}{4\left(\omega_{1}-E_{r}+E_{\widetilde{r}}\right)}\left(\sin 4 \phi_{r} X_{k}^{\bar{r} r}-\sin ^{2} 2 \phi_{r}\left(X_{k}^{r r}-X_{k}^{\overline{r r}}\right)\right)_{\omega_{2}-\omega_{1}} \\
& -\sum_{r} \frac{\delta_{k, l} d_{s}{ }^{2}}{4\left(\omega_{1}+E_{r}-E_{\tilde{r}}\right)}\left(\sin 4 \phi_{r} X_{k}^{r \bar{r}}-\sin ^{2} 2 \phi_{r}\left(X_{k}^{r r}-X_{k}^{\overline{r r}}\right)\right)_{\omega_{2}-\omega_{1}} .
\end{aligned}
$$

Retaining only the pseudospin contributions to the polarizability operator means that we consider Raman scattering on the local anharmonic vibrations. Due to the electron transfer this scattering is modified by the effective retarded interaction between pseudospins and by the electron interband transitions. The terms in the expression for $\left\{\left\{M_{k}^{\gamma} \mid M_{l}^{\gamma}\right\}\right\}$ which are of the higher order with respect to the parameter $t_{i j}$ describe the electron and mixed contributions to the Raman scattering tensor.

We shall restrict ourself in this work to the pseudospin Raman scattering. Basing on expression (17) we write for the polarizability operator

$$
\hat{P}_{k_{2},-k_{1}}^{\gamma \gamma}\left(\omega_{1}, t\right)=-2 \pi \sum_{n} e^{i\left(k_{2}-k_{1}\right) R_{n}} \int_{-\infty}^{+\infty} d \omega^{\prime} e^{i\left(\omega_{1}-\omega^{\prime}\right) t}\left\{\left\{\hat{M}_{n}^{\gamma} \mid \hat{M}_{n}^{\gamma}\right\}\right\}_{\omega^{\prime}, \omega_{1}}
$$

the scattering tensor can be rewritten as:

$$
H_{k_{2}, k_{1}}^{\gamma \gamma, \gamma \gamma}\left(\omega_{1}, \omega_{2}\right)=\frac{(2 \pi)^{2}}{\left(e^{\beta \omega}-1\right)} \sum_{n, n_{1}} e^{i\left(n-n_{1}\right)\left(k_{2}-k_{1}\right)} 2 \operatorname{Im}\left\langle\left\langle\left\{\left\{M_{n}^{\gamma} \mid M_{n}^{\gamma}\right\}\right\}_{\omega_{2}, \omega_{1}} \mid\left\{\left\{M_{n_{1}}^{\gamma} \mid M_{n_{1}}^{\gamma}\right\}\right\}_{-\omega_{2},-\omega_{1}}\right\rangle\right\rangle_{\omega+i \epsilon}
$$

where $\omega=\omega_{2}-\omega_{1}$. Here the correlation functions $\langle X(t) X\rangle$ are expressed in terms of two-time Green's functions.

\section{SCATTERING INTENSITY IN THE CASE OF ZERO ELECTRON TRANSFER}

Let us consider at first a more simple case of the absence of electron transfer $(t=0)$ when the light scatters on the noninteracting pseudospins. Using expression (19) for the scattering tensor and taking into account that in this situation the functions $\left\langle\left\langle X_{n_{1}}^{r \tilde{r}} \mid X_{n}^{\tilde{r} r}\right\rangle\right\rangle_{\omega_{2}-\omega_{1}}=\left\langle\left\langle X_{n_{1}}^{\widetilde{r} r} \mid X_{n}^{r \widetilde{r}}\right\rangle\right\rangle_{\omega_{1}-\omega_{2}}$ are different from zero only, we obtain:

$$
H_{k_{2}, k_{1}}^{\gamma \gamma, \gamma \gamma}\left(\omega_{1}, \omega_{2}\right) \equiv H_{k_{2}, k_{1}}\left(\omega_{1}, \omega_{2}\right)=\frac{(2 \pi)^{2} d_{s}{ }^{4}}{16\left(e^{\beta \omega}-1\right)} \sum_{r, n, n_{1}} e^{i\left(\left(\mathbf{k}_{2}-\mathbf{k}_{1}\right) \mathbf{R}_{n}-\left(\mathbf{k}_{2}-\mathbf{k}_{1}\right) \mathbf{R}_{n_{1}}\right)}
$$




$$
\begin{aligned}
& \times \sin ^{2} 4 \phi_{r}\left(2 \operatorname{Im}\left\langle\left\langle X_{n_{1}}^{r \tilde{r}} \mid X_{n}^{\tilde{r} r}\right\rangle\right\rangle_{\omega_{2}-\omega_{1}}\left(\frac{1}{\omega_{1}}-\frac{1}{\omega_{1}+\lambda_{r}-\lambda_{\tilde{r}}}\right)^{2}\right. \\
& \left.+2 \operatorname{Im}\left\langle\left\langle X_{n_{1}}^{\tilde{r} r} \mid X_{n}^{r \tilde{r}}\right\rangle\right\rangle_{\omega_{2}-\omega_{1}}\left(\frac{1}{\omega_{1}}-\frac{1}{\omega_{1}-\lambda_{r}+\lambda_{\tilde{r}}}\right)^{2}\right) .
\end{aligned}
$$

Since at $t=0$

$$
\left\langle\left\langle X_{n_{1}}^{r \tilde{r}} \mid X_{n}^{\tilde{r} r}\right\rangle\right\rangle_{\omega_{2}-\omega_{1}}=\frac{\delta_{n n_{1}}\left\langle X^{r r}-X^{\widetilde{r} \widetilde{r}}\right\rangle}{\omega_{2}-\omega_{1}+\lambda_{r}-\lambda_{\tilde{r}}}
$$

the expression for the scattering tensor can be written as:

$$
\begin{aligned}
H_{k_{2}, k_{1}}\left(\omega_{1}, \omega_{2}\right) & =\frac{N(2 \pi)^{2} d_{s}^{4}}{16} \sum_{r} \sin ^{2} 4 \phi_{r} \\
& \times\left(\left\langle X^{\widetilde{r r}}\right\rangle \delta\left(\omega_{2}-\omega_{1}+\lambda_{r}-\lambda_{\bar{r}}\right)\left(\frac{1}{\omega_{1}}-\frac{1}{\omega_{1}+\lambda_{r}-\lambda_{\widetilde{r}}}\right)^{2}\right. \\
& \left.+\left\langle X^{r r}\right\rangle \delta\left(\omega_{2}-\omega_{1}-\lambda_{r}+\lambda_{\widetilde{r}}\right)\left(\frac{1}{\omega_{1}}-\frac{1}{\omega_{1}-\lambda_{r}+\lambda_{\tilde{r}}}\right)^{2}\right)
\end{aligned}
$$

where

$$
\begin{aligned}
& \left\langle X^{\widetilde{r} \tilde{r}}\right\rangle=\frac{e^{-\beta \lambda_{\tilde{r}}}}{\sum_{r}\left(e^{-\beta \lambda_{\tilde{r}}}+e^{-\beta \lambda_{r}}\right)}, \\
& \left\langle X^{r r}\right\rangle=\frac{e^{-\beta \lambda_{r}}}{\sum_{r}\left(e^{-\beta \lambda_{\tilde{r}}}+e^{-\beta \lambda_{r}}\right)} .
\end{aligned}
$$

In this case the Raman scattering spectrum consists of delta-peaks corresponding to the transitions $r \leftrightarrow \widetilde{r}$ between energy levels of pseudospin interacting with electrons. Energy of the pseudospin flip depends on the occupancy of the electron states in the same unit cell. This is the reason for the splitting in the Raman spectrum.

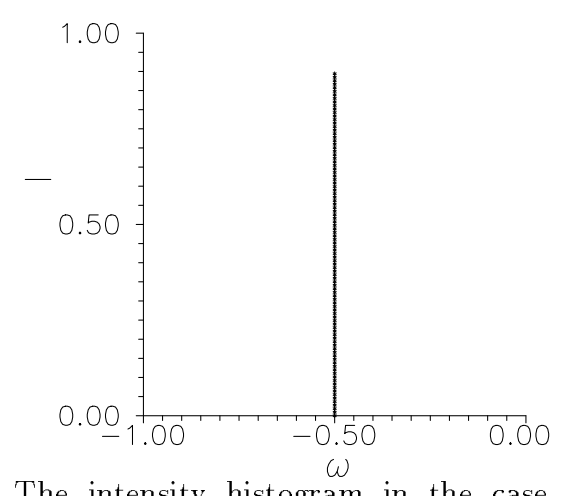

Fig. 1. The intensity histogram in the case $\lambda_{\tilde{1}}<\lambda_{\tilde{2}}$, $\lambda_{\tilde{1}}<\lambda_{\tilde{3}}\left(\mu<\mu_{1}, T=0\right) . I=\sin ^{2} 4 \phi_{1}\left\langle X^{\tilde{1} \tilde{1}}\right\rangle \delta\left(\omega-\lambda_{\tilde{1}}+\lambda_{1}\right)$, $g=1, \Omega=0.4, U=4, h=0.3[14]$.
In the regime of the fixed value of the chemical potential ( $\mu=$ const) we can observe in the limit $T \rightarrow 0$ only one delta-peak for the Raman intensity which corresponds to the frequency change connected with the transition from the lowest energy level $|\tilde{r}\rangle$ to the excited one $\mid r>$ ( in the case of Stokes component of the scattering) For instance, when $\lambda_{\tilde{1}}<\lambda_{\tilde{3}}, \lambda_{\tilde{1}}<\lambda_{\tilde{2}}$, the frequency change is $\omega_{2}-\omega_{1}=\lambda_{\tilde{1}}-\lambda_{1}=-\sqrt{h^{2}+\Omega^{2}}$, the intensity is proportional to $\sin ^{2} 4 \phi_{1}$ (Fig. 1). In general, if $\lambda_{\tilde{r}}$ is the lowest energy, the frequency change is equal to $\lambda_{\tilde{r}}-\lambda_{r}=-\sqrt{\left(n_{r} g-h\right)^{2}+\Omega^{2}}$ (where $n_{r}=0, r=$ $1 ; n_{r}=1, r=3,4 ; n_{r}=2, r=2$ ), the intensity is proportional to $\sin ^{2} 4 \phi_{r}$.

The diagram illustrating the ground state of the system depending on the values of the field $h$ and chemical potential $\mu$ is presented in Fig. 2. Border lines between the regions with different ground states are given by the conditions

$$
\begin{aligned}
\mu & =\mu_{1} \equiv \frac{1}{2}\left(\sqrt{h^{2}+\Omega^{2}}\right. \\
& \left.-\sqrt{(g-h)^{2}+\Omega^{2}}\right), \quad \lambda_{\overline{1}}=\lambda_{\tilde{3}, \overline{4}} \\
\mu & =\mu_{2} \equiv \frac{1}{2}\left(\sqrt{(g-h)^{2}+\Omega^{2}}\right. \\
& \left.-\sqrt{(2 g-h)^{2}+\Omega^{2}}\right)+U, \quad \lambda_{\tilde{3}, \overline{4}}=\lambda_{\tilde{2}} .
\end{aligned}
$$




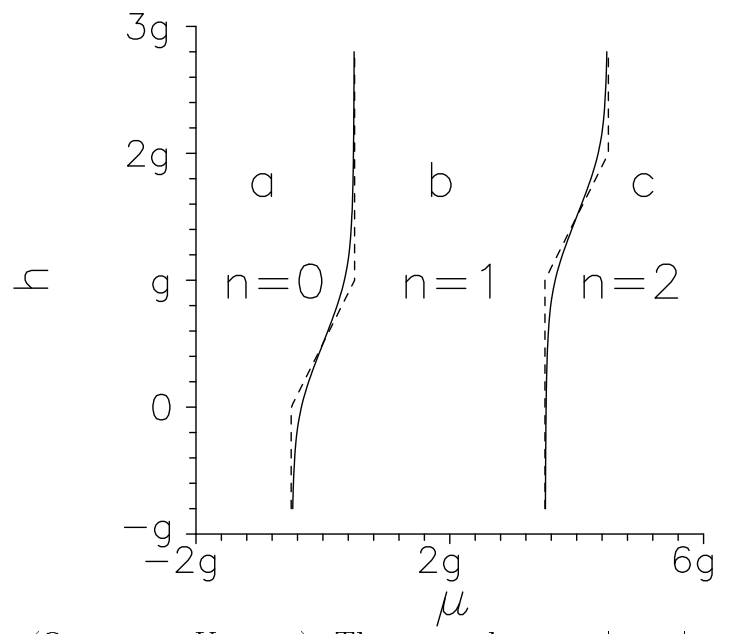

Fig. 2. The ground state diagram. $(\Omega=0.4 g, U=4 g)$. The ground states $|1>| 3>,(\mid 4>)$ and $\mid 2>$ correspond to the regions $a, b$, and $c$ respectively. Dashed lines refer to the case $\Omega=0$.

At zero temperature the electron concentration $n=\left\langle\sum_{\sigma} n_{i \sigma}\right\rangle$ jumps between $n=0$ and $n=1$ at $\mu=\mu_{1}$ and between $n=1$ and $n=2$ at $\mu=\mu_{2}$.

The Raman spectral lines at frequencies $\omega^{\prime}=-\sqrt{h^{2}+\Omega^{2}}, \omega^{\prime \prime}=-\sqrt{(g-h)^{2}+\Omega^{2}}, \omega^{\prime \prime \prime}=-\sqrt{(2 g-h)^{2}+\Omega^{2}}$ exist at $T=0$ in the regions a, b, c of the ground state diagram (Fig. 2), respectively. They coexist only on the border lines. It follows that in the $n=$ const regime the Raman spectrum has two peaks situated at the $\omega^{\prime}$ and $\omega^{\prime \prime}$ frequencies in the case $0<n<1$ and at the $\omega^{\prime \prime}$ and $\omega^{\prime \prime \prime}$ frequencies in the case $1<n<2$. The intensities of peaks redistribute in each pair at the change of the electron concentration (see Fig. 3).
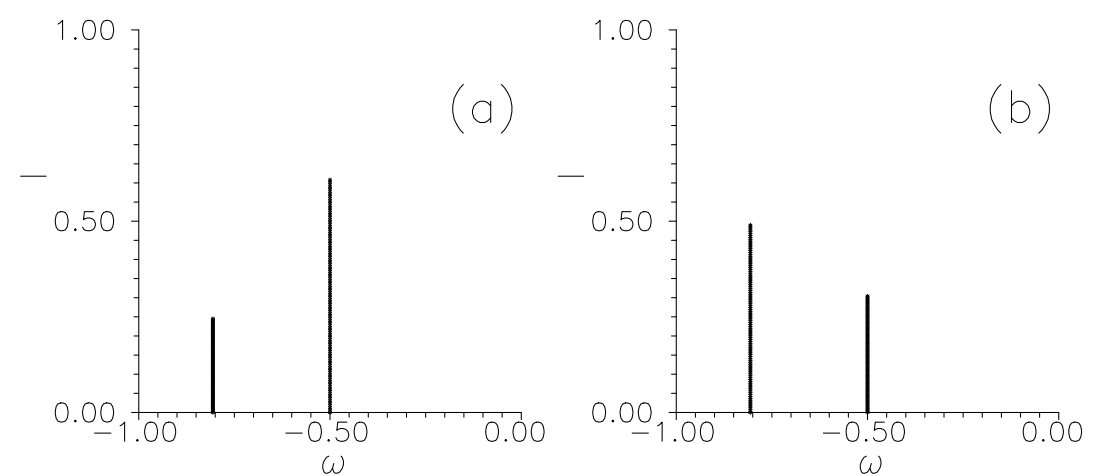

Fig. 3. The intensity histogram $\left(n=\right.$ const, $\left.T=0, \lambda_{\tilde{1}}=\lambda_{\tilde{3}}\right) . I=\sum_{i=1,3,4} \sin ^{2} 4 \phi_{i}\left\langle\underset{X^{i i}}{\omega} \delta\left(\omega-\lambda_{i}+\lambda_{i}\right), g=1, \Omega=0.4, U=4\right.$, $h=0.3, n=0.3$ (a), $n=0.6(\mathrm{~b})$.

Full spectrum consisting of three Stokes lines can be observed only at $T \neq 0$. The intensities of additional delta-peaks which appear besides the main ones are exponentially small being proportional to $e^{-\beta\left(\lambda_{\tilde{p}}-\lambda_{\tilde{r}}\right)}$.

\section{POLARIZABILITY GREEN'S FUNCTION IN GRPA}

Now we shall take into account the possibility of the electron hopping from site to site. Under the influence of electron transfer the positions and shapes of lines in the Raman scattering spectrum can change essentially. It is our aim to investigate this question .
We consider as an example one of the lines: the line with the frequency $\omega_{2}-\omega_{1}=\lambda_{\overline{1}}-\lambda_{1} \equiv \omega^{\prime}$ which is the lowest in magnitude and can be observed at the fixed electron concentration with the value $0<n<1$. The calculation of the correlators $\left\langle X^{p q}(t) X^{r s}\right\rangle$ (with $p q, r s=11, \tilde{1} \tilde{1}, 1 \widetilde{1}, \widetilde{1} 1)$ entering expression (2) for the Raman scattering tensor will be performed on the basis of the temperature Green's function method using the perturbation theory and diagram technique for Hubbard operators. The general random phase approximation elaborated for calculation of Bose-correlators in the case of Fermi-systems with strong short-range correlations [11] will be used. Such an approach was applied in [12] at the investigation of dielectric susceptibility of 
the pseudospin-electron model in the zero tunneling case $(\Omega=0)$.

The Hamiltonian (6) is presented as

$$
\begin{aligned}
& H=H_{0}+H_{i n t}, \\
& H_{0}=\sum_{i, r} \lambda_{r} X_{i}^{r r}, \\
& H_{i n t}=\sum_{i, j, \sigma} t_{i, j} \hat{c}_{i, \sigma}^{\dagger} \hat{c}_{j, \sigma} .
\end{aligned}
$$

We consider temperature Green's function

$$
\begin{aligned}
\left.\left\langle T X_{l}^{r s}(\tau) \mid X_{m}^{p q}(\tau)\right\rangle\right\rangle & =K_{l m}^{p q r s}\left(\tau-\tau^{\prime}\right) \\
& =\left\langle T X_{l}^{r s}(\tau) X_{m}^{p q}(\tau) \sigma(\beta)\right\rangle_{0}^{c}, \\
\sigma(\beta) & =T \exp \left(-\int_{0}^{\beta} H_{i n t}(\tau) d \tau\right),
\end{aligned}
$$

the operators are written in the interaction representation: $C(\tau)=e^{\tau H_{0}} C e^{-\tau H_{0}}$, the brackets $\langle\ldots\rangle_{0}$ stand for the statistical averaging with the Hamiltonian $H_{0}$. The symbol $\langle\ldots\rangle^{c}$ is used for the separation of connected diagrams.

Expanding the exponent in the powers of $H_{\text {int }}$, we obtain the averages of $T$-products of Hubbard operators.
To calculate such averages one can use Wick's theorem for Hubbard operators [15]. In accordance with this theorem the average of product of $n X$ operators can be reduced to the sum of the averages of products of the $n-1$ operators. After this procedure the result is expressed in terms of nonperturbated Green's functions

$$
\begin{aligned}
g_{i j}^{p q}\left(\tau-\tau^{\prime}\right) & =\delta_{i j} g^{p q}\left(\tau-\tau^{\prime}\right) \\
& =\delta_{i j} \frac{\left.\left\langle T X^{p q}(\tau) \mid X^{r s}\left(\tau^{\prime}\right)\right\rangle\right\rangle}{\left\langle\left[X^{r s}, X^{p q}\right]_{ \pm}\right\rangle_{0}}
\end{aligned}
$$

the Fourier transform of which is given by the expression

$$
\begin{aligned}
g^{p q}(\omega) & =\int_{0}^{\beta} d\left(\tau-\tau^{\prime}\right) e^{-i \omega_{n}\left(\tau-\tau^{\prime}\right)} g^{p q}\left(\tau-\tau^{\prime}\right) \\
& = \pm \frac{1}{i \omega_{n}-\lambda_{p q}}
\end{aligned}
$$

here the upper (lower) sign corresponds to Green's functions constructed on Fermi- (Bose-) type operators; $\omega_{n}=\frac{2 n+1}{\beta} \pi$ or $\omega_{n}=\frac{2 n}{\beta} \pi$ respectively. The remaining averages of the products of diagonal $X$-operators are expanded in terms of semi-invariants [15].

The following transformation of correlators $K_{l m}\left(\tau-\tau^{\prime}\right)$ to the momentum-frequency representation is used:

$$
\int_{0}^{\beta} d\left(\tau-\tau^{\prime}\right) e^{i \omega_{n}\left(\tau-\tau^{\prime}\right)} \frac{1}{N} \sum_{l m} K_{l m}^{p q r s}\left(\tau-\tau^{\prime}\right) e^{i q\left(R_{l}-R_{m}\right)}=K^{p q r s}\left(q, \omega_{n}\right) .
$$

At the diagrammatic representation of the terms of the perturbation series for correlators $K\left(q, \omega_{n}\right)$ we use the standard notations []: triangle $\quad$ pq stands for Green's function $g^{p q}$; circles denote the averages $\left\langle X^{p p} \pm X^{q q}\right\rangle_{0}$ and $\left\langle X^{p p}\right\rangle_{0}$, respectively; oval surrounding a certain number $(l)$ of circles denotes the semi-invariant $\left\langle X^{p_{1} p_{1}} \ldots X^{p_{l} p_{l}}\right\rangle_{0}^{c}$ of the $l$-th order; the wavy line $\backsim \sim$ is an electron transfer $t_{i j}$.

It is known that the infinite sum of chain-like fragments consisting of Fermi functions $g^{p q}$ bound consecutively by the lines $t_{i j}$ corresponds to Green's function calculated in the Hubbard-I approximation. Let us introduce the notation

$\mathcal{G}^{p q}=$

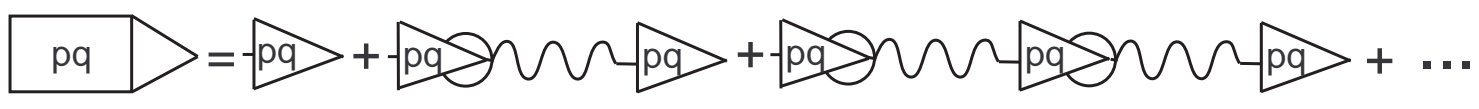

or in the analytic form

$$
\mathcal{G}^{p q}=\frac{1}{i \omega_{n}-\epsilon_{p q}(k)}, \epsilon_{p q}(k)=\lambda_{p q}+A_{p q}\left\langle X^{p p}+X^{q q}\right\rangle t_{k}
$$


here $\lambda_{p q}=\lambda_{p}-\lambda_{q} ; t_{k}=\sum_{i-j} t_{i j} e^{i R\left(R_{i}-R_{j}\right)}, A_{\widetilde{41}}=\cos ^{2} \phi_{1}$, $A_{\overline{4} 1}=\sin ^{2} \phi_{1}$. The formula (31) corresponds to the particular case of the Hubbard-I approximation, namely to the so-called independent subbands approach. It is suitable when the distance between Hubbard subbands is much larger than their widths.

The transitions with the pseudospin reorientation at the partial filling of electron states $(0<n<1)$ are responsible for the Raman scattering which comes of the scattering with the frequency $\omega^{\prime}$ at $t_{i j}=0$. The electron subbands $\lambda_{\tilde{4} 1}=\lambda_{\tilde{3} 1}, \lambda_{\tilde{4} \tilde{1}}=\lambda_{\tilde{3}}$ taking part in these transitions are situated far from others and are sufficiently separated between themselves, when the fol- lowing inequalities are fulfilled: $U, g>>h, \Omega>W$ ( $W=\sum_{j} t_{i j}$ ). In this case we can use the approximation (31) leaving under consideration only the states $|1\rangle,|\widetilde{1}\rangle$, $|\widetilde{3}\rangle$ and $|\widetilde{4}\rangle$.

At the summation of diagrams for correlators $K^{\text {pqrs }}\left(q, \omega_{n}\right)$ we restrict ourselves in the spirit of GRPA to the diagrams having a structure of multi-loop chains. The simplest diagrams of this type for $(p, q, r, s)=(1, \tilde{1})$ are given in the Appendix. It is shown that the main contributions in the considered frequency region $\left(\omega \sim \omega^{\prime}\right)$ are connected with $K^{1 \widetilde{111}}$ correlator.

The diagrammatic series for $K^{1111}$ can be presented in the following compact form:

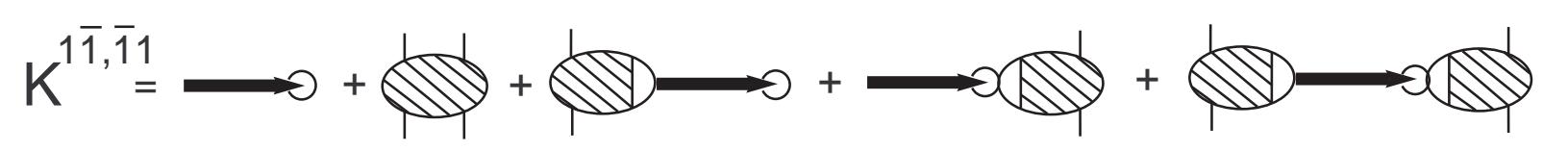

The first term is "full" boson Green's function which satisfies the Dyson equation

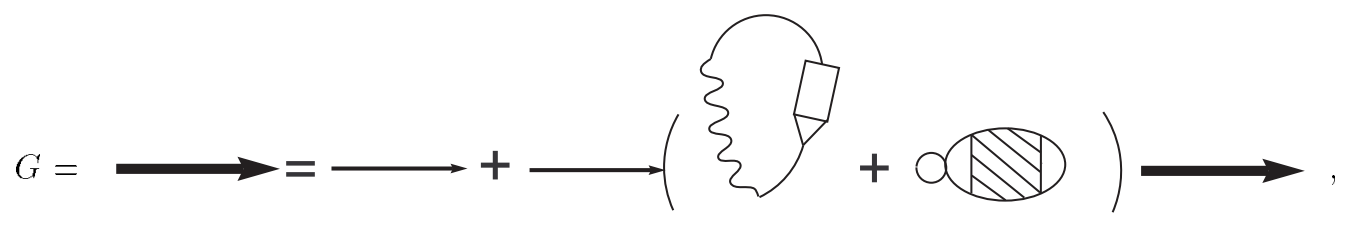

where the thin arrow stands for Bose-type nonperturbated Green's function $g^{1 \overline{1}}$, given by the expression (29). The shaded ellipses denote the multi-loop diagrams which consist of products of the following zero-order loops:
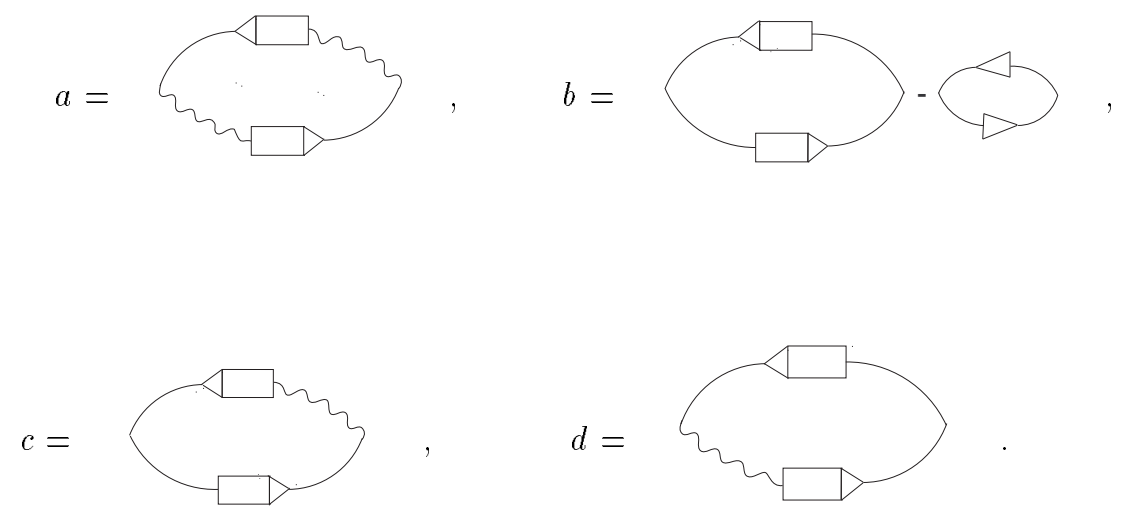

or in the simple symbolic form:

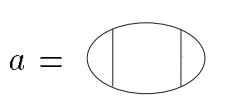<smiles>CC1CC(C)C(C)C(C)CC1C</smiles><smiles>C=CC1CC2CCC(CC2C)C1C</smiles><smiles>CC1C2CCCC1C2=O</smiles>

The junctions between the zero-order loops are realized in the following way: 

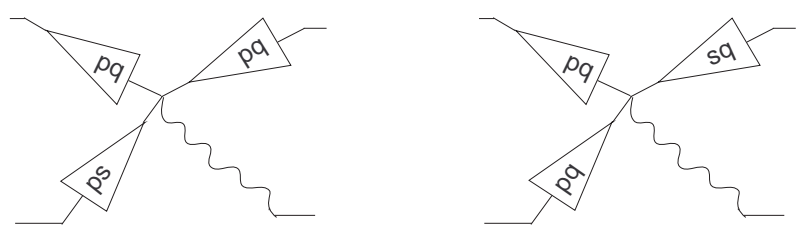

We do not consider junctions by semi-invariants because they lead to the appearance of delta-function $\delta(\omega)$ [12], and corresponding diagrams do not contribute to the Raman scattering intensity.

As is shown in the Appendix, the most important of the zero-order loop diagrams are

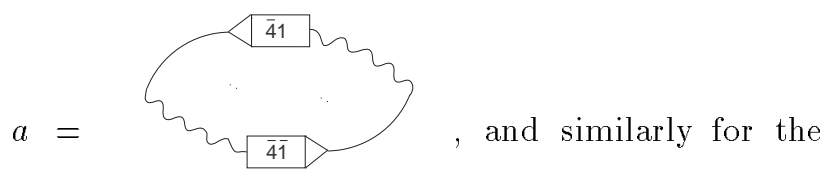

loops $b, c, d$ (the lower Green's function is $\widetilde{\mathcal{G}^{41}}$ or $\mathcal{G}^{\widetilde{31}}$, the upper one is $\mathcal{G}^{\overline{4} 1}$ or $\mathcal{G}^{\overline{3} 1}$ ); in the analytic form:

$$
\begin{aligned}
& a=\frac{-2 \sin ^{2} \phi_{1} \cos ^{2} \phi_{1}}{N} \sum_{k} t_{k} t_{k+q} \frac{n\left(\epsilon_{\widetilde{41}}(k)\right)-n\left(\epsilon_{4 \tilde{1}}(k+q)\right)}{i \omega_{n}+\epsilon_{\widetilde{41}}(k)-\epsilon_{\tilde{4} 1}(k+q)} \\
& b=\frac{-2}{N} \sum_{k} \frac{n\left(\epsilon_{\widetilde{41}}(k)\right)-n\left(\epsilon_{\widetilde{4} 1}(k+q)\right)}{i \omega_{n}+\epsilon_{\widetilde{41}}(k)-\epsilon_{\tilde{4}}(k+q)}+2 \frac{n\left(\lambda_{\widetilde{41}}\right)-n\left(\lambda_{\tilde{4} 1}\right)}{i \omega_{n}+\lambda_{\widetilde{41}}-\lambda_{\tilde{4} 1}} \\
& c=\frac{-2 \cos ^{2} \phi_{1}}{N} \sum_{k} t_{k} \frac{n\left(\epsilon_{\widetilde{41}}(k)\right)-n\left(\epsilon_{\widetilde{4} 1}(k+q)\right)}{i \omega_{n}+\epsilon_{\widetilde{41}}(k)-\epsilon_{\widetilde{4} 1}(k+q)} \\
& d=\frac{-2 \sin ^{2} \phi_{1}}{N} \sum_{k} t_{k+q} \frac{n\left(\epsilon_{\widetilde{41}}(k)\right)-n\left(\epsilon_{\widetilde{4} 1}(k+q)\right)}{i \omega_{n}+\epsilon_{\widetilde{41}}(k)-\epsilon_{\tilde{4} 1}(k+q)} \\
& q=k_{2}-k_{1}
\end{aligned}
$$

(we consider a paramagnetic state and electron spectrum is degenerated with respect to electron spin).

The summation of the diagrammatic series for the multi-loop diagrams is equivalent to the solving of the following set of simultaneous equations (see [12])

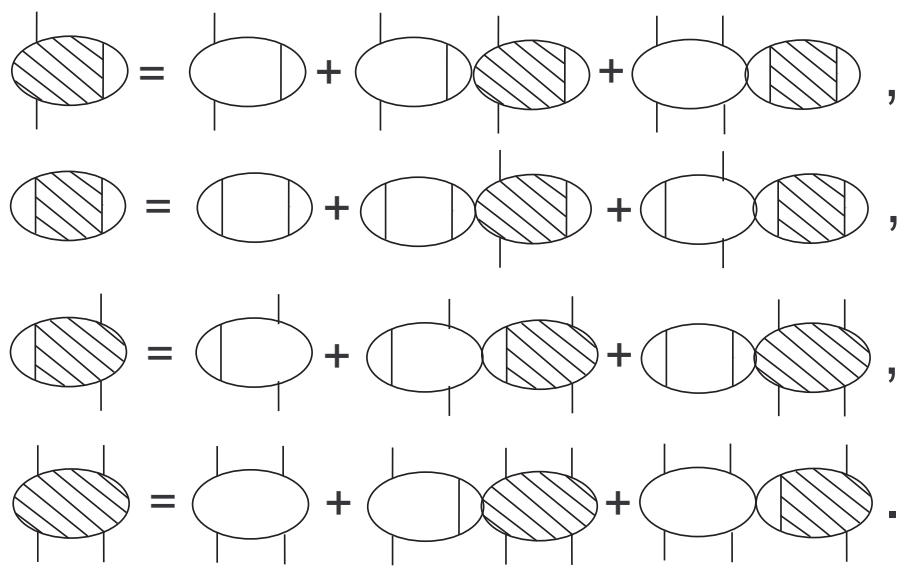

It's formal solution is

$$
\begin{aligned}
& A=\infty=\frac{a}{1-c-d+c d-a b}, \quad B=\quad=\frac{b}{1-c-d+c d-a b}, \\
& C=N=\frac{c-c d+a b}{1-c-d+c d-a b}, \quad D=W=\frac{d-c d+a b}{1-c-d+c d-a b} .
\end{aligned}
$$

Let us now turn to "full" Green's function $G$. The analytic expression for this function following from the written above Dyson equation is: 


$$
G=\frac{\left\langle X^{\tilde{11}}-X^{11}\right\rangle}{i \omega_{n}+\lambda_{1}-\lambda_{\tilde{1}}+\frac{2}{N} \sum_{k} t_{k}\left[n\left(\epsilon_{\tilde{4} 1}\right) \sin ^{2} \phi_{1}-n\left(\epsilon_{\overline{41}}\right) \cos ^{2} \phi_{1}\right]-A\left\langle X^{\tilde{11}}-X^{11}\right\rangle} .
$$

The self-energy part (a mass operator) of the function $G$ consists of two terms. The first of them has a form of an loop insertion into the line of boson Green's function and leads to the renormalization

$$
\lambda_{r} \rightarrow \widetilde{\lambda_{r}}=\lambda_{r}+\frac{1}{N} \sum_{k, p} A_{p r} t_{k} n\left(\epsilon_{p}(k)\right)
$$

of "bare" energy levels of non-perturbated problem in the spirit of mean field corrections (it would be a true mean field at the substitution $\langle X\rangle_{0} \rightarrow\langle X\rangle$ in the loop contribution). Such a renormalization, if it is taken into account, should be made everywhere, in particular, in the expression for Fermi-type Green's function $\mathcal{G}^{p q}[16]$.

The second term in a self-energy part of $G$ function is frequency and momentum dependent and describes an effective retarded interaction through band electrons between pseudospins. It plays here the same role as a Fourier-transform $J(q)$ in the model with direct interaction $J_{i j} S_{i}^{z} S_{j}^{z}$ (in this last case expression (33) corresponds to the summation of the so-called chain diagrams when the expansion in powers of the reciprocal radius of interaction is used).

The expression (33) is analogous in its structure to the obtained one in [11] for the transverse magnetic susceptibility of the Hubbard model. The formulae for magnon Green's function in $s-d$ exchange model (see [17]), when the effective interaction between spins via conducting electrons is taken into account within the usual RPA scheme is also a simplified analogue of (33). In contrast to these examples we have to deal here with a split spectrum. The contribution connected with interband transitions are especially actual in our case.

Formulae (32) and (33) give the possibility to write in the explicit form the pseudospin correlator $K^{1 \widetilde{111}}$, using the expression

$$
K^{1 \widetilde{111}}=G+B+G D+G C+G D C
$$

which follows from the diagrammatic representation.

The transition to the time correlation function $\left\langle X^{11}(t) X^{11}(0)\right\rangle$ can be performed in a standard way [18]:

$$
\begin{aligned}
\left\langle X^{\tilde{1} 1}(t) X^{1 \widetilde{1}}(0)\right\rangle & =\frac{1}{2 \pi} \int_{-\infty}^{\infty} d \omega \frac{e^{i \omega t}}{e^{\beta \omega}-1} \\
& \times\left[-2 \operatorname{Im}\left\langle\left\langle X^{1 \tilde{1}} \mid X^{\tilde{1} 1}\right\rangle\right\rangle_{\omega+i \epsilon}\right]
\end{aligned}
$$

$$
\left\langle\left\langle X^{1 \overline{1}} \mid X^{\overline{1} 1}\right\rangle\right\rangle_{\omega}=K^{1 \overline{111}}\left(i \omega_{n} \rightarrow \omega\right)
$$

\section{PSEUDOSPIN RAMAN SCATTERING INTENSITY}

The results obtained in the previous section at the calculation of the pseudospin correlator $K^{1 \widetilde{111}}$ allow to write the expression for the Raman scattering tensor in GRPA

$$
\begin{aligned}
H_{k_{2}, k_{1}}\left(\omega_{1}, \omega_{2}\right) & =\frac{(2 \pi)^{2} d^{4}}{16\left(e^{\beta \omega}-1\right)} \sin ^{2} 4 \phi_{1}\left(\frac{1}{\omega_{1}}-\frac{1}{\omega_{1}+\epsilon_{r}}\right)^{2} \\
& \times 2 \operatorname{Im}(G+B+G D \\
& +G C+G D C)_{\omega+i \epsilon}
\end{aligned}
$$

and to investigate the influence of electron transfer on the frequency dependence of the Raman scattering intensity (which is described by the effective cross-section of the scattering).

It should be mentioned that in accordance with the approximations adopted above formula (38) concerns the scattering at frequencies which are close to the $\omega_{2}-\omega_{1}=$ $\omega^{\prime}$ line (it is the smallest in magnitude one of the scattering frequency lines in the case of the zero electron transfer and exists at the less than half filling of electron states $(0<n<1))$.

In the case of low temperatures $(T \rightarrow 0)$ we can carry out integration in the expressions for the zero-order loops (32) in the analytic form putting $\mathbf{q}=\mathbf{k}_{2}-\mathbf{k}_{1}=0$ (what is justified by inequality $\frac{2 \pi}{k_{1,2}} \gg a$, where $a$ is lattice constant) and using the rectangular density of states

$$
\rho(t)=\left\{\begin{array}{ll}
\frac{1}{2 W}, & -W \leq t \leq W \\
0, & \text { otherwise }
\end{array} .\right.
$$

The following expressions are obtained

$$
\begin{aligned}
b & =\left.\frac{\ln \left|\omega+t\left(\cos ^{2} \phi_{1}-\frac{n}{2}\right)\right|}{W\left(\cos ^{2} \phi_{1}-\frac{n}{2}\right)}\right|_{-W \frac{1-\frac{3}{2} n}{1-\frac{\pi}{2}}} ^{W} \\
& -\frac{i \pi}{W\left|\cos ^{2} \phi_{1}-\frac{n}{2}\right|}-2\left(\frac{1}{\omega}-i \pi \delta(\omega)\right) \theta\left(\frac{2}{3}-n\right),
\end{aligned}
$$




$$
\begin{aligned}
& c=\left.\frac{\cos ^{2} \phi_{1} t}{W\left(\cos ^{2} \phi_{1}-\frac{n}{2}\right)}\right|_{-W \frac{1-\frac{3}{2} n}{1-\frac{n}{2}}} ^{W}+\frac{\cos ^{2} \phi_{1} \omega}{\cos ^{2} \phi_{1}-\frac{n}{2}} b, \\
& d=c \frac{\sin ^{2} \phi_{1}}{\cos ^{2} \phi_{1}}, \\
& a=\left.\frac{\cos ^{2} \phi_{1} \sin ^{2} \phi_{1} t^{2}}{2 W\left(\cos ^{2} \phi_{1}-\frac{n}{2}\right)}\right|_{-W \frac{1-\frac{3}{2} n}{1-\frac{n}{2}}} ^{W}+\frac{\cos ^{2} \phi_{1} \sin ^{2} \phi_{1} \omega}{\cos ^{2} \phi_{1}-\frac{n}{2}} c .
\end{aligned}
$$

Here we have replaced $i \omega_{n} \rightarrow \omega+i \epsilon$; the frequency $\omega$ is counted off from the $\omega^{\prime}=\lambda_{\tilde{1}}-\lambda_{1}$ value. The connection between chemical potential and electron concentration is taken into account [10]; in the case of two electron subbands $\epsilon_{\widetilde{41}}$ and $\epsilon_{\widetilde{4} 1}$

$$
\mu=\lambda_{\overline{4}}-\lambda_{\overline{1}}-W \cos ^{2} \phi_{1}\left(1-\frac{3}{2} n\right) .
$$

It follows from this relation that chemical potential is placed in the upper subband; the positions of subband edges and the chemical potential depending on electron concentration are shown in Fig. 4, the widths of subbands are $\left(1-\frac{n}{2}\right) \cos ^{2} \phi_{1} W$ and $\frac{n}{2} \sin ^{2} \phi_{1} W$, respectively.

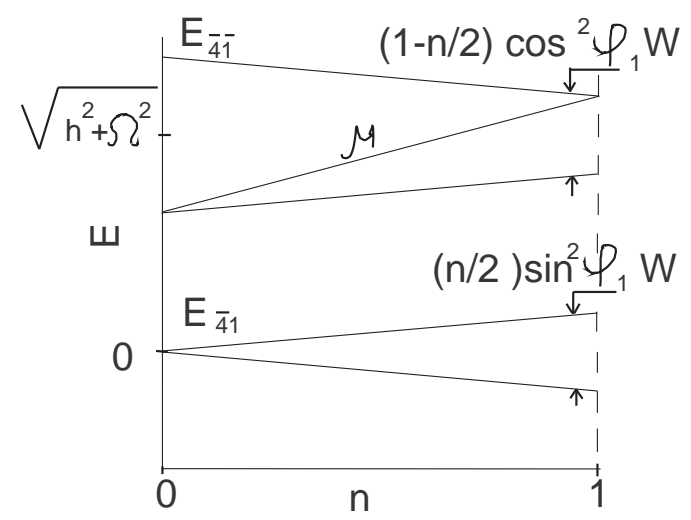

Fig. 4. Band boundaries and chemical potential for $T=0$.

The mean field type renormalization (34) of energies of pseudospin states is not taken into account here and in the subsequent numerical calculations (this effect is not essential from the point of view of frequency dependence of scattering intensity but can manifest itself in the dependence of Raman spectrum on the electron concentration).

The imaginary parts of all loop contributions are different from zero only in the frequency interval

$$
-W \frac{1-\frac{3}{2} n}{1-\frac{n}{2}}<-\frac{\omega}{\cos ^{2} \phi_{1}-\frac{n}{2}}<W
$$

The same is valid for the multi-loop contributions.
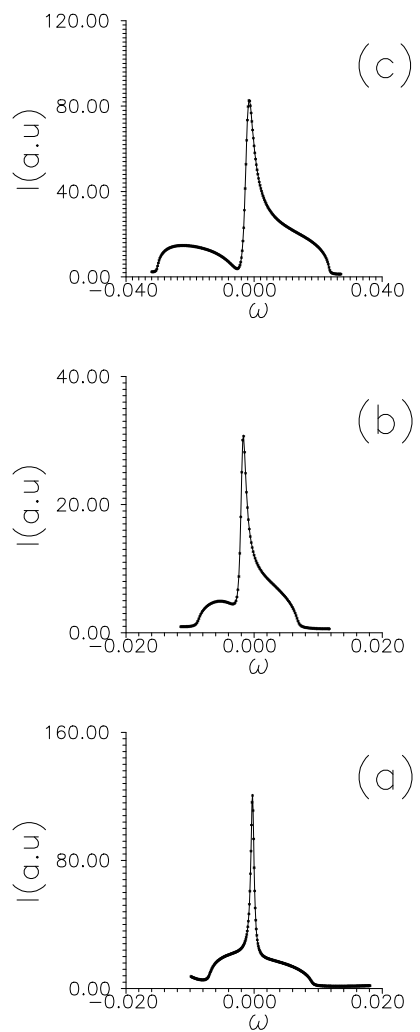

Fig. 5. Raman scattering intensity for $T=0, W=0.1$, $n=0.2$, a) $h=0.5, \Omega=0.1$; b) $h=0.4, \Omega=0.5$; c) $h=0.1$, $\Omega=0.5$.
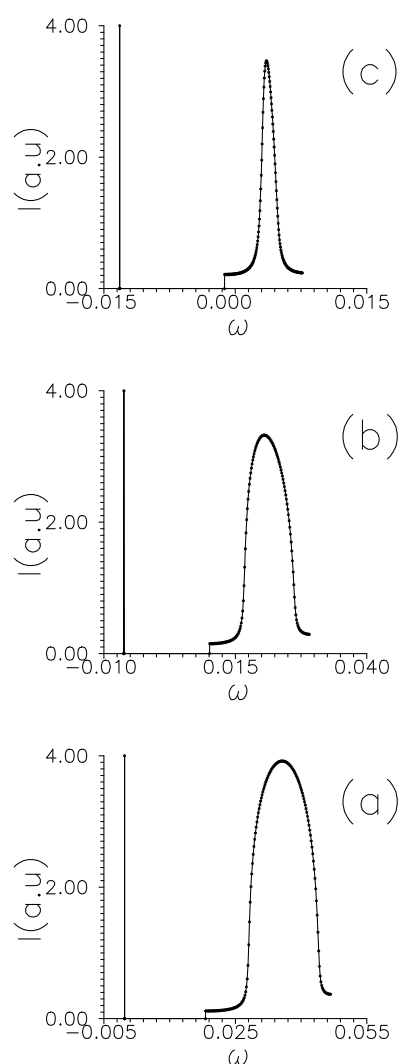

Fig. 6. Raman scattering intensity for $T=0, W=0.1$, $n=0.9$, a) $h=0.5, \Omega=0.1$; b) $\mathrm{h}=0.4, \Omega=0.5$; c) $h=0.1$, $\Omega=0.5$. The vertical line in each graph schematically shows the location of delta-peak. 
The imaginary part of boson Green's function $G$ in the frequency region (42) is formed by the function $A(\omega+i \epsilon)$ and is finite. It can also have the character of delta-peak

$$
\operatorname{Im} G(\omega+i \epsilon)=-\pi \frac{\left\langle X^{\overline{11}}-X^{11}\right\rangle}{F^{\prime}(\widetilde{\omega})} \delta(\omega-\widetilde{\omega})
$$

outside this interval; here $\widetilde{\omega}$ is a root of the equation $F(\omega)=0$, where

$$
F(\omega)=\omega-\operatorname{Re} A(\omega)\left\langle X^{\tilde{1} \tilde{1}}-X^{11}\right\rangle
$$

The fulfilment of conditions

$$
\frac{-\widetilde{\omega}}{\cos ^{2} \phi_{1}-\frac{n}{2}}>W \text { or } \frac{-\widetilde{\omega}}{\cos ^{2} \phi_{1}-\frac{n}{2}}<-W \frac{1-\frac{3}{2} n}{1-\frac{n}{2}}
$$

is necessary in this case. It realizes, as can be seen, at large electron concentrations.

As a result, at $T=0$ the full contribution to the Raman scattering tensor consists of two components: (a) the relatively broad band the width of which $\Delta \omega=\frac{4(1-n)}{2-n} W$ changes with the change of $n$ (from $\Delta \omega=2 W$ at $n=0$ to $\Delta \omega=0$ at $n=1$ ); (b) the narrow peak that at large electron concentrations is in the shape of the $\delta$-function while at small ones is over-lapped by the above mentioned band and is smeared.

The first of these components has an incoherent character. It is connected with interband transitions from the occupied states of the $\lambda_{\overline{4} 1}$ subband to the unoccupied ones of the $\lambda_{\overline{41}}$ subband and reflects the reconstruction of electron states at the reorientation of the pseudospin. The second component is coherent by nature having a pure pseudospin origin. It expresses the collective pseudospin dynamics that is formed by the effective interaction between pseudospins via conducting electrons $(\tilde{\omega}$ is a limit value of the pseudospin wave frequency at $\mathbf{q}=0$ ).

The calculations of the Raman scattering intensity profiles $I(\omega) \sim H_{k_{2}, k_{1}}$ at $T=0$ have been performed numerically on the basis of formulae (33) and (38). Their results at the various values of $h$ and $\Omega$ parameters [14] as well as at different electron concentrations are given in Fig. 5, 6. The separate existence of coherent (a $\delta$-peak) and incoherent (a band) parts of the Raman spectrum at large electron concentrations $(n=0,9)$ and their overlapping at small concentrations $(n=0,2)$ is illustrated. At the small $n$ values the intensity curves possess presumably asymmetric shape with the wing on the large frequencies side.

The decrease of the full intensity of the Raman scattering at the increase of electron concentration is a characteristic feature of the obtained scattering profiles. This fact is in agreement with the behaviour of the $\omega_{2}-\omega_{1}=$ $\omega^{\prime}$ line, which is responsible for the considered type of scattering at the zero electron transfer (namely, the scattering with the pseudospin flips on the sites unoccupied by electrons; the number of such sites tends to zero at $n \rightarrow 1$ ).
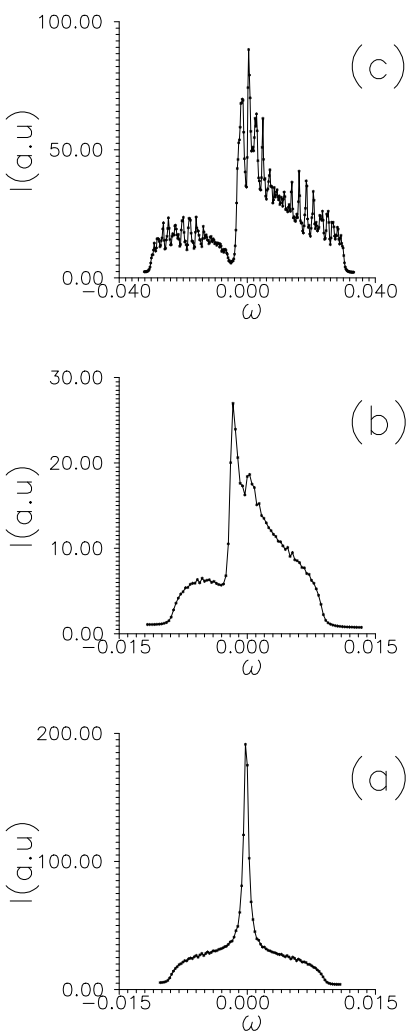

Fig. 7. Raman scattering intensity for $T=0.005, W=0.1$, $n=0.2$, a) $h=0.5, \Omega=0.1$; b) $h=0.4, \Omega=0.5$; c) $h=0.1$, $\Omega=0.5$.
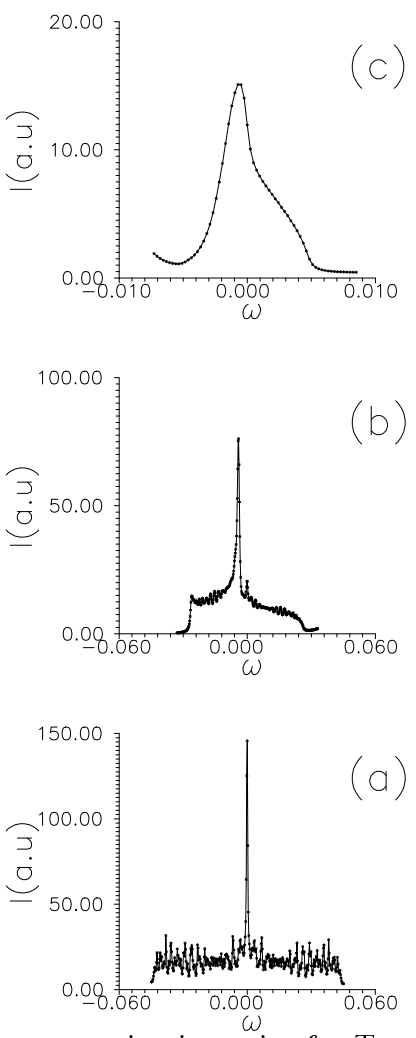

Fig. 8. Raman scattering intensity for $T=0.005, W=0.1$, $n=0.9$, a) $h=0.5, \Omega=0.1$; b) $h=0.4, \Omega=0.5$; c) $h=0.1$, $\Omega=0.5$. 


\section{V. STASYUK, T. S. MYSAKOVYCH}

At the temperatures different from zero the incoherent band in the scattering spectrum becomes broader occupying the interval $-W<\frac{-\omega}{\cos ^{2} \phi_{1}-n / 2}<W$, because now the interband transitions between states with any energies are possible due to the smearing of Fermi distribution functions $n\left(\lambda_{\widetilde{41}}\right)$ and $n\left(\lambda_{\tilde{4} 1}\right)$. This band can cover the $\widetilde{\omega}$ frequency at all values of electron concentration creating a sufficiently sharp but diffuse peak.

The results of the Raman scattering profile calculations at $T \neq 0$, performed with the help of formula (38) by means of the direct integration of expressions (32) over the wave vector, are presented in Fig. 7, 8. At small concentrations ( $n=0,2$, Fig. 7.) the intensity curves differ only slightly from the corresponding curves at $T=0$ (Fig. 5.), but at large concentrations $(n=0,9$, Fig. 8.) the difference is more significant. This is connected with the above mentioned overlapping of the $\delta$-like coherent peak with the continuous band at $T \neq 0$ in the whole concentration region. The general structure of scattering spectra at the small and large electron concentrations becomes similar.

\section{CONCLUSIONS}

In this work the investigation of the Raman scattering spectrum in the pseudospin-electron model with the strong short-range electron correlation of the Hubbard type is performed. The microscopic approach based on the operator expansion scheme at the construction of the polarizability operator of a system is used. The contributions connected with the scattering on the pseudospin excitations in the presence of the interaction with conducting electrons are considered. The case of the zero electron transfer is investigated separately; the scattering is caused here by the dipole transitions connected with the pseudospin reorientations only (i.e. the pure anharmonic phonon scattering). The peculiarities of the frequency dependence of the Raman scattering intensity, that appear due to electron transfer and are caused by the transitions between electron subbands, are revealed.

It is established that the Raman scattering line consists of a coherent ( $\delta$-peak like) and incoherent (in the form of a band of the finite width) components which can be separated (at large electron concentrations and low temperatures) or mutually overlap (at small values of $n$ or at the increase of $T$ ). This result is a reflection of the collective dynamics of the pseudospin wave type as well as of the dynamical reconstruction of the electron spectrum at the reorientation of pseudospins. An analogue of this picture can be found in the Hubbard and $t-J$ models: one can separate in the transverse magnetic susceptibility the contributions from localized and collectivized (having a band origin) magnetic moments [11].

The form of lines and their width depend on the electron concentration and temperature; the asymmetry of lines is their characteristic feature. The intensity profiles are similar to those which are obtained in the case of scattering on the antiferromagnetic magnons, but their asymmetry is more appreciable having a form that is closer to the observed experimentally one in the $\mathrm{YBa}_{2} \mathrm{Cu}_{3} \mathrm{O}_{7-\delta}$ crystals [4]. It should be pointed out, however, that the consistent analysis of experimental data from the point of view of possible role of pseudospins in the scattering can be performed after the supplementary study of the pseudospin dynamics influence on the magnon scattering (as it was shown in [19], the pseudospin-electron coupling leads to the modification of the effective exchange interaction and has, therefore, an effect on the magnon spectrum).

\section{APPENDIX}

We shall demonstrate here the structure of diagrammatic series appearing at the calculation of temperature Green's function constructed of the polarizability operators on the examples of typical diagrams of the second order. In the adopted approximation the polarizability operator $\hat{P}_{k_{2}-k_{1}}$ is expressed in terms of the operators $X^{1 \widetilde{1}}, X^{\widetilde{11}}, X^{11}-X^{\widetilde{11}}$ and is a linear function of them. Therefore, we consider the correlator $K_{l m}^{p q^{r s}}\left(\tau-\tau^{\prime}\right)$ with the indices $(p q, r s)$ belonging to the subspace $(1, \widetilde{1})$. In particular, the following diagrams appear: in the case of the function $\left\langle T X_{l}^{1 \widetilde{1}}(\tau) X_{m}^{\overline{11}}\left(\tau^{\prime}\right)\right\rangle$ :
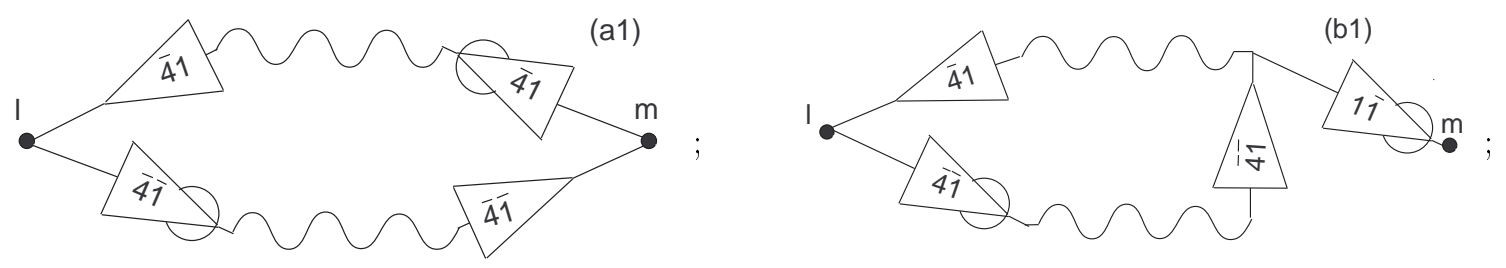


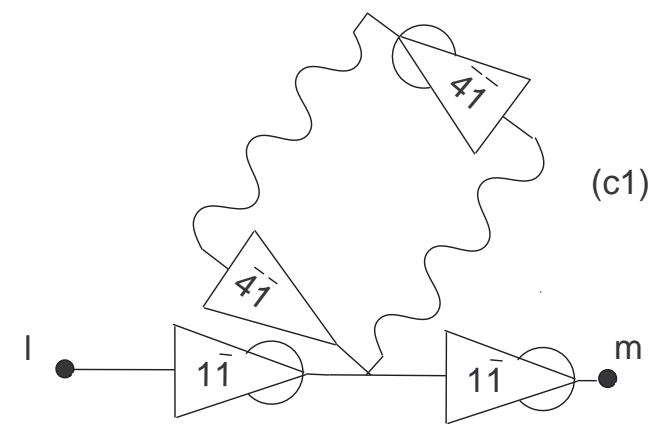

(d1)

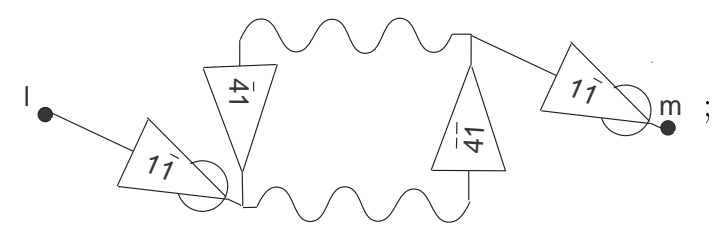

in the case of the functions $\left\langle T X_{l}^{11}(\tau) X_{m}^{\widetilde{11}}\left(\tau^{\prime}\right)\right\rangle,\left\langle T X_{l}^{\widetilde{11}}(\tau) X_{m}^{\widetilde{11}}\left(\tau^{\prime}\right)\right\rangle$ :
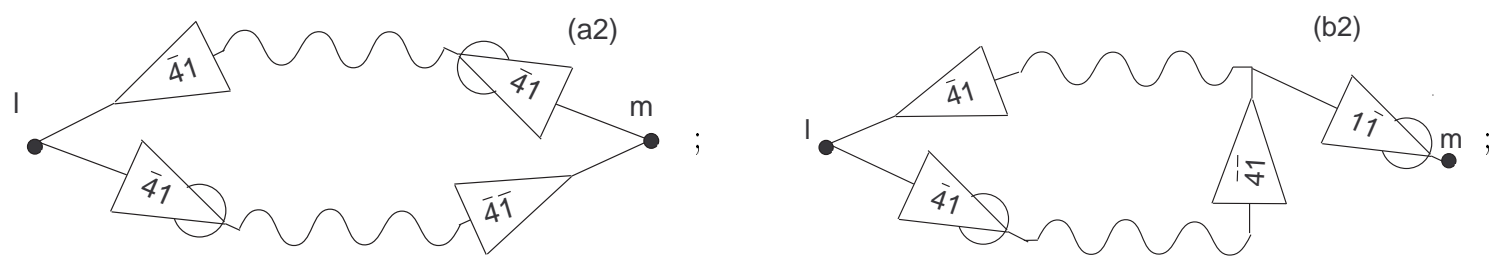

(c2)

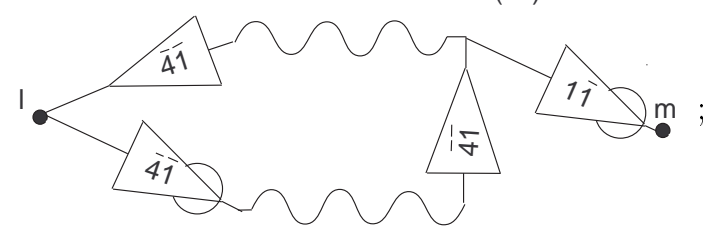

in the case of the functions $\left\langle T X_{l}^{\widetilde{11}}(\tau) X_{m}^{11}\left(\tau^{\prime}\right)\right\rangle,\left\langle T X_{l}^{11}(\tau) X_{m}^{11}\left(\tau^{\prime}\right)\right\rangle$ :

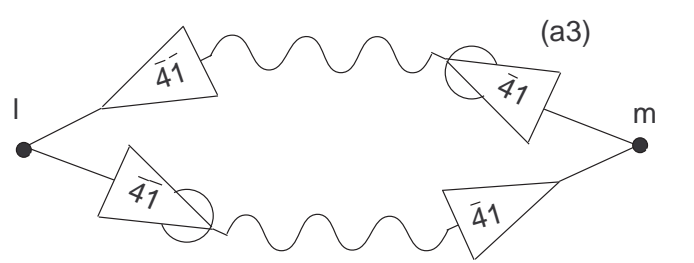

(c3)

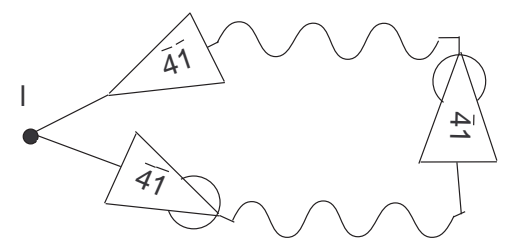

(11)

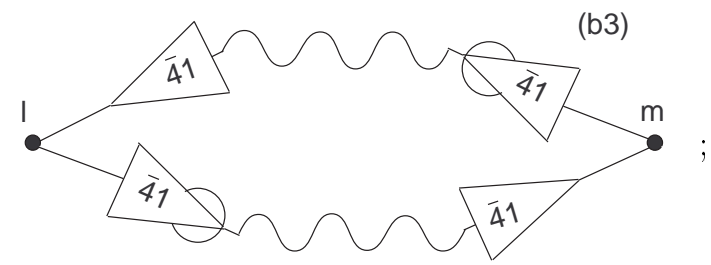

(d3)
We have shown here among others diagrams (c3), (d3) having at the left or right ends the diagonal $X$-operators (which are denoted by circles). At the subsequent averaging with the help of expansion in terms of semi-invariants and at the connection with other diagram fragments (in the case of the higher order diagrams) by means of semiinvariants such a type of diagrams give contributions pro- portional to $\delta(\omega)$. Such terms are not taken into account, because they do not lead to the change of light frequency at the scattering and as a result do not contribute to the Raman scattering effect. This does not concern, however, the diagram (c2) as well as the diagrams of the higher order with similar fragments at the left or right ends, where the connection by semi-invariants is absent. 


\title{
I. V. STASYUK, T. S. MYSAKOVYCH
}

The chain fragments, in which the states of the first and the last Green's function differ in the pseudospin orientation, lead due to the identity

$$
\frac{1}{x-a} \frac{1}{x-b}=\left(\frac{1}{x-a}-\frac{1}{x-b}\right) \frac{1}{a-b}
$$

to the appearance of the factors $\frac{t}{\lambda_{\tilde{4} \tilde{1}}-\lambda_{\tilde{4} 1}}=\frac{t}{\sqrt{h^{2}+\Omega^{2}}}$ in the contributions of the diagrams (a2), (b2), (a3). In accordance with the approximation used in our consideration such a type of diagrams can be referred to the higher order of smallness.

It should be mentioned also that diagrams with the same pseudospin orientation in both parts of the loop (ones of (c2), (b3) type) describe the intraband scattering (the scattering frequency is of the $\omega=\omega_{2}-\omega_{1} \sim W$ order). In the case when pseudospin reorientate at the both ends of the loop ((a1), (b1), (c1), (d1) diagrams), the scattering has an interband character $\left(\omega \sim \epsilon_{\tilde{4} 1}-\epsilon_{\tilde{41}} \sim\right.$ $\left.\lambda_{\tilde{1}_{1}}\right)$. The last frequency region is the subject of our investigation.

Because of that we restrict ourselves to the consideration of the loop diagrams of (a1), (b1), (c1), (d1) type.
[1] M. V. Klein, in Light Scattering in Solids, edited by M. Cardona (Springer-Verlag, Berlin-HeidelbergNewYork, 1975)

[2] P. Fleury, R. Loudon, Phys. Rev. 166, 514 (1968).

[3] B. S. Shastry, B. I. Shraiman, Phys. Rev. Lett. 65, 1068 (1990).

[4] M. Pressl, M. Mayer, P. Knoll, S. Lo, U. Hohenster, J. Raman Spectroscopy 27, 343 (1996).

[5] I. V. Stasyuk, T. S. Mysakovych, preprint ICMP-9828E, Lviv (1998)

[6] R. A. Cowley, Adv. Phys. 12, 421 (1963).

[7] R. Barry, I. W. Sharpe, Can. J. Phys. 56, 550 (1978).

[8] I. V. Stasyuk, Ya. l. Ivankiv, preprint ITP-87-57P, Kyiv (1987).

[9] K. A. Müller, Z. Phys. B, 80, 193 (1990).

[10] I. V. Stasyuk, A. M. Shvaika, Phys. C 213, 57 (1993).

[11] Yu. A. Izyumov, B. M. Letfulov, J. Phys.: Cond. Matt. 2, 8905 (1990).

[12] I. V. Stasyuk, A. M. Shvaika, Cond. Matt. Phys. iss. 3, 134 (1993).
[13] I. V. Stasyuk, T. S. Mysakovych, preprint ICMP-9834E, Lviv (1998).

[14] The relative parameter values correspond to the typical ones for the systems described by PEM, see [20,21].

[15] P. M. Slobodyan, I. V. Stasyuk, Teor. Mat. Fiz. 19, 423 (1974).

[16] I. V. Stasyuk, A. M. Shvaika, K. V. Tabunshchyk, Cond. Matt. Phys. 2, 109 (1999).

[17] Yu. A. Izyumov, F. A. Kassan-ogly, Yu. N. Skryabin, Polievyie mietody $v$ tieorii ferromagnietizma (Field methods in the theory of ferromagnetism) (Наука, Москва, 1974).

[18] D. N. Zubarev, Usp. Fiz. Nauk 71, 71 (1960).

[19] I. V. Stasyuk, A. M. Shvaika, Acta Phys. Pol. A, 84, 293 (1993).

[20] M. Frick, W. von der Linden, I. Morgenstern, H. Raedt, Z. Phys. B 81, 327 (1990).

[21] N. M. Plakida, V. S. Udovenko, Mod. Phys. Lett. B6, $541(1992)$

\section{КОМБІНАЩІӤНЕ РОЗСІЯННЯ В ПСЕВДОСПІН-ЕЛЕКТРОННІЙ МОДЕЛІ}

\author{
I. В. Стасюк, Т. С. Мисакович \\ Інститут фізики конденсованих систем Національной академї наук Украӥни, \\ вул. Свенціиького, 1, Львів, 79011, Украӥна \\ E-mail: ista@icmp.lviv.ua
}

Дослілжено ангармонічні фононні (псевдоспінові) внески до комбінапійного розсіяння світла у кристалах із сильною короткосяжною електронною кореляпією, що описуються псевдоспін-електронною моделлю. Для розрахунку компонент тензора розсіяння використано мікроскопічний підхід, який грунтується на операторному розкладі за степенями константи електронного переносу при побудові оператора поляризованости. У рамках узагальненого наближення хаотичних фаз ураховано вплив ефективної взаємодії між псевдоспінами через електрони провідности. Вивчено особливості колективної динаміки псевдоспінів та виділено складові спектра розсіяння, пов'язані зі збудженнями псевдоспін-хвильового типу, а також із реконструкцією електронного спектра при реорієнтації псевдоспінів. Проаналізовано частотну залежність інтенсивности комбінаційного розсіяння світла при різних значеннях електронної концентрації та температури. 